\title{
SEROPREVALENCIA DE LEPTOSPIROSIS EN ALPACAS DE QUIMSACHATA, PUNO
}

\author{
Seroprevalence of Leptospirosis in Alpacas From \\ Quimsachata, Puno
}

\author{
Yván Santos S. ${ }^{1}$, Francisco Suárez A. ${ }^{1,2}$, Hermelinda Rivera G. ${ }^{3}$, Wilfredo Huanca L. ${ }^{4}$, \\ Oscar Cárdenas M. ${ }^{5}$ y José Camacho S. $^{6}$
}

\section{Resumen}

El objetivo del presente estudio fue determinar la seroprevalencia de leptospirosis en alpacas durante la época de lluvias (enero-marzo) en la localidad Quimsachata, Puno. Se analizaron 344 sueros de alpacas de una estación experimental del Instituto Nacional de Investigación y Extensión Agraria, mediante la prueba de microaglutinación. Se evaluó, además, la asociación de las variables sexo y edad con la reacción a la prueba. El44.8 $\pm 5.3 \%$ de los animales fueron serorreactores. Los serovares detectados fueron pomona (43.6\%), icterohaemorrhagiae (9.9\%), ycanicola (1.5\%), no encontrándose ningún reactor a hardjo. No se encontró ningún suero positivo en tuis; mientras que en adultos no se encontró diferencia significativa entre sexos. Los altos títulos hallados para pomona e icterohaemorrhagiae en estos animales sugieren una infección activa durante la época de lluvia.

Palabras clave: leptospirosis, alpacas, seroprevalencia

\section{Abstract}

The objective of this study was to estimate the prevalence of leptospirosis in alpacas reared in the locality of Quimsachata, Puno during the rainy season (January-March). Serum samples $(n=344)$ of alpacas from the experimental station of the Instituto Nacional de Investigación y Extensión Agraria (INIA) were analyzed using the microaglutination test. The association of age and sex with test results was also evaluated. The overall seroprevalence was $44.8 \pm 5.3 \%$. The detected serovars were pomona $(43.6 \%)$, icterohaemorrhagiae (9.9\%), canicola (1.5\%), and none for hardjo.Positive samples in young animals («tuis») were nil, whereas in adult animals no statistical differences was found due to sex. The high antibody titres inpomona andicterohaemorrhagiae suggested an active infection during the rainy period.

Key words: leptospirosis, alpacas, seroprevalence

${ }^{1}$ Laboratorio de Medicina Veterinaria Preventiva, ${ }^{3}$ Laboratorio de Microbiología y Parasitología Veterinaria, ${ }^{4}$ Laboratorio de Reproducción Animal, ${ }^{6}$ Laboratorio de Zootecnia y Producción Agropecuaria, Facultad de Medicina Veterinaria, Universidad Nacional Mayor de San Marcos, Lima

${ }^{3}$ E-mail: francisco_suarez2001@hotmail.com

${ }^{5}$ Programa Nacional de Investigación en Camélidos, ILLPA-INIA, Puno 


\section{INTRODUCCIÓN}

La leptospirosis es una zoonosis de distribución mundial que afecta tanto al hombre como a los animales domésticos y silvestres. Se han descrito más de 220 serovares a nivel mundial, pero mayormente, las infecciones se producen por un número limitado de serovares endémicos de una región o país, y su presencia está íntimamente ligada a factores ecológicos y medioambientales (Thiermann, 1984). La leptospirosis es considerada una enfermedad infecciosa emergente, ya que constituye un problema de $\mathrm{Sa}$ lud Pública en muchos países en desarrollo por su alta letalidad (Levett, 2001).

La enfermedad se adquiere por contacto directo cutáneo o ingestión de aguas contaminadas, por orina de animales infectados, sangre y tejidos de reservorios que atraviesan las mucosas y la piel a través de abrasiones o pequeñas heridas, o por el contacto directo con los reservorios. La importancia de esta enfermedad depende del número de microorganismos infectantes, de las defensas inmunológicas del hospedador, de la serovariedad causal y de la virulencia de la cepa (Elizalde et al., 2004).

El mantenimiento del agente en la naturaleza depende, fundamentalmente, del prolongado periodo de bacteriuria de los animales portadores y de la capacidad de supervivencia del microorganismo en el medio ambiente. El pH alcalino del suelo, la baja radiación solar, el clima templado y las inundaciones, son factores que favorecen su supervivencia y diseminación a través de las aguas superficiales contaminadas (Acha y Szyfres, 2003). La epidemiología de la leptospirosis ha sido modificada por cambios en la agricultura, animales, clima y comportamiento humano (Martínez et al., 1998; Levett, 2001).

En las últimas décadas se han intensificado los estudios sobre los aspectos productivos de los camélidos sudamericanos a fin de impulsar su desarrollo en economías re- gionales alternativas. Este desarrollo requiere optimizar la eficiencia reproductiva, para lo cual es necesario conocer tanto los parámetros fisiológicos como la susceptibilidad y la respuesta inmune de estas especies ante los agentes infecciosos. Dentro de las enfermedades infecciosas que pueden afectar la reproducción, la leptospirosis es una de las patologías probables (Johnson, 1989).

En el Perú los trabajos sobre leptospirosis en camélidos sudamericanos son pocos (Ludeña y Vargas, 1982; Macedo y Hung, 1993; Herrera et al., 2000; Rosadio et al., 2003). En Argentina se han reportado los serovares pomona, grippotyphosa y patoc en llamas, guanacos y vicuñas (Brihuega et al., 1996; Llorente et al., 2002), en tanto que en un zoológico de Cuba en animales silvestres se encontró que el serogrupo pomona fue el más frecuente en varias especies afectadas (Sosa et al., 1988).

La leptospirosis se encuentra ampliamente difundida en el Perú. Ha sido reportada como causa de epizootias en bovinos y porcinos; y se le ha encontrado serológicamente en diversas especies de animales de producción y de compañía, por lo que se requiere conocer su situación actual en el país ya que no hay estudios actualizados (Liceras de Hidalgo et al., 1989). El presente estudio tuvo como objetivo evaluar la presencia de anticuerpos contra leptospiras en sueros de alpacas de una empresa alpaquera del sur del Perú, durante la época de lluvias, y su asociación con la edad y sexo de los animales.

\section{Materiales y Métodos}

\section{Lugar de Estudio y Animales}

La toma de muestras se llevó a cabo durante los meses de enero a marzo de 2003 en el Centro de Investigación y Producción Quimsachata de la Estación Experimental ILLPA del Instituto Nacional de Investigación Agropecuaria (INIA), ubicada en el 
distrito de Santa Lucía, departamento de Puno, a una altitud de $4300 \mathrm{msnm}$. La zona tiene, en promedio, una temperatura mínima de $-4.7^{\circ} \mathrm{C}$ y una máxima de $13.2^{\circ} \mathrm{C}$, y una humedad relativa de $55 \%$.

El tamaño mínimo de muestra de 317 animales se calculó empleando la fórmula para estimar una proporción para poblaciones finitas (Daniel, 1996). Para esto, se utilizó un nivel de confianza de 0.95 , una proporción referencial de 0.5 y un error máximo admisible de 0.05 . La población total era de 1800 animales, donde 293 eran tuis machos, 230 tuis hembras, 204 adultos machos y 1073 adultos hembras. Se llegó a trabajar con 344 alpacas, las cuales fueron proporcionalmente estratificadas según la edad y el sexo (Pérez, 2000). Se consideró como alpaca tui desde el destete hasta los dos años y como alpaca adulta a los mayores de dos años de edad.

\section{Muestras y Análisis de Laboratorio}

Las muestras de sangre se colectaron por punción de la vena yugular y se centrifugaron por 10 minutos a $3000 \mathrm{rpm}$. Los sueros resultantes se guardaron a $-20^{\circ} \mathrm{C}$ hasta su traslado al Laboratorio de Microbiología de la Facultad de Medicina Veterinaria de la Universidad Nacional Mayor de San Marcos, en Lima, donde se procesaron mediante la técnica de microaglutinación (MAT).

Para esto, se prepararon diluciones seriadas de las muestras de suero junto con suero fisiológico, desde 1:50 hasta 1:1600. Se colocaron en microplacas de 96 pozos y el antígeno de Leptospira se sembró en cada uno de los pozos. Se incubó por 2 horas a 28 ${ }^{\circ} \mathrm{C}$ y la lectura se hizo en un microscopio de campo oscuro, comparando los resultados de las muestras problema con el control positivo (antígeno) y negativo (suero fisiológico). Se utilizó cepas de leptospiras canicola, icterohaemorrhagiae, pomona y hardjo y se consideró como sueros positivos a aquellos que den títulos mayores o iguales a 1:100 (Myers, 1985).

\section{Análisis de Datos}

Los resultados fueron expresados como porcentaje de prevalencia (Sentís et al., 1995) con sus respectivos intervalos de confianza. Además, se analizó la asociación del evento en estudio (reactores a leptospirosis) con las variables consideradas (sexo y edad) mediante la prueba no paramétrica de Chi cuadrado (Berquó et al., 1981).

\section{Resultados}

De 344 muestras de sueros analizados, $154(44.8 \pm 5.3 \%)$ fueron positivos a anticuerpos contra leptospiras, correspondiendo a los serovares pomona, icterohaemorrhagiae y canicola, no encontrándose ningún reactor a hardjo. El Cuadro 1 muestra las frecuencias de serovares detectados en los animales, observándose que el más frecuente fue pomona con $43.6 \%$ (150/344). La mayoría de los sueros positivos a leptospirosis reaccionaron frente a un solo serovar (119), mientras que 34 sueros reaccionaron a dos serovares, y un suero dio positivo a tres serovares, dando un total de $10.2 \%$ de serorreacción múltiple.

La frecuencia de animales positivos en alpacas adultas machos fue de $74.4 \pm 13.7 \%$ en tanto que en adultos hembras fue $61.0 \pm$ $6.7 \%$, sin haber diferencia estadística entre sexos. No se encontraron muestras positivas en tuis (Cuadro 2). El título más alto de los sueros positivos en el caso de canicola fue de 1:200, para icterohaemorrhagiae de 1:6400 y para pomona de 1:12800.

\section{Discusión}

La mayor seroprevalencia correspondió al serovar pomona (43.6\%), en forma similar a otros reportes en la sierra sur del país y con ligeras variaciones en los otros serovares (Herrera et al., 2000; Rosadio et al., 2003). Cabe destacar que el muestreo 
Cuadro 1. Frecuencia $\left(\% \pm \mathrm{IC}^{1}\right)$ de serovariedades de Leptospira detectadas en alpacas $(\mathrm{n}=$ 344) del Centro de Investigación y Producción Quimsachata del Instituto Nacional de Investigación Agropecuaria (INIA), mediante la prueba de microaglutinación (Puno, 2003) ${ }^{2}$

\begin{tabular}{lcc}
\hline Serovar & $\mathrm{N}^{\circ}$ de alpacas positivas & Positivos $(\%) \pm \mathrm{IC}$ \\
\hline Pomona & 150 & $43.6 \pm 5.2$ \\
Icterohaemorrhagiae & 34 & $9.9 \pm 3.2$ \\
Canicola & 5 & $1.5 \pm 1.3$ \\
\hline
\end{tabular}

1 Intervalo de confianza del $95 \%$

2 Se encontró 154 alpacas serorreactoras de un total de 344 , donde 35 animales dieron positivo a más de un serovar

Cuadro 2. Distribución de alpacas según sexo y edad, y su reacción a leptospirosis mediante la prueba de microaglutinación, en el C.I.P. Quimsachata del Instituto Nacional de Investigación Agropecuaria (INIA) (Puno, 2003)

\begin{tabular}{|c|c|c|c|}
\hline Tipo de animal & $\mathrm{N}^{\circ}$ de alpacas & $\mathrm{N}^{\circ}$ de reactores & Positivos $(\%) \pm \mathrm{IC}$ \\
\hline \multicolumn{4}{|l|}{ Tuis } \\
\hline Machos & 56 & 0 & 0 \\
\hline Hembras & 44 & 0 & 0 \\
\hline \multicolumn{4}{|l|}{ Adultos } \\
\hline Machos & 39 & 29 & $74.4 \pm 13.7$ \\
\hline Hembras & 205 & 125 & $61.0 \pm 6.7$ \\
\hline Total & 344 & 154 & $44.8 \pm 5.3$ \\
\hline
\end{tabular}

en estos estudios anteriores se realizó en época de lluvias, y se sabe que las leptospiras son dependientes de condiciones ambientales óptimas para su supervivencia y desarrollo. Las condiciones ambientales y climáticas de las zonas altoandinas donde habitan los camélidos sudamericanos corresponden predominantemente a un clima seco, con temperaturas extremas, de lluvias estacionales y por lo general escasas. Al respecto, Herrera et al. (2000) señalan que las lluvias funcionan como un factor condicionante para la infección por leptospiras, afirmando que la ocu- rrencia de leptospirosis en alpacas se debe a una etiología multifactorial. Por otro lado, la presencia de otras especies en el hábitat de los camélidos podría contribuir a que la enfermedad esté presente durante todo el año (Liceras de Hidalgo et al., 1989).

La ausencia de sueros positivos en animales jóvenes (tuis) es contraria a lo que se menciona sobre leptospirosis, donde la enfermedad es más severa en terneros ocasionando una disminución del desarrollo y una tasa de mortalidad variable; además, la 
serovariedad pomona es un patógeno porcino donde el vacuno es un hospedador accidental (Radostits et al., 2002; Acha y Szyfres, 2003). Por otro lado, los ovinos no son hospedadores de mantenimiento naturales de pomona y hardjjo, y suelen tener infecciones de duración relativamente corta (Radostits et al., 2002).

La prevalencia de leptospirosis en las alpacas evaluadas mediante la prueba de hemaglutinación fue de $44.8 \%$, correspondiendo la mayor frecuencia al serovar pomona (43.6\%), seguido de icterohaemorrhagiae $(9.9 \%)$, corroborando los hallazgos de Rosadio et al. (2003), quienes reportan las mismas serovariedades, aunque con distintas frecuencias (19\% para icterohaemorrhagiae y $47 \%$ para canicola). Cabe destacar que la fase de campo se realizó en la época de lluvias, que da condiciones óptimas para la supervivencia y desarrollo de la leptospira. No obstante, los camélidos sudamericanos de las zonas altoandinas se encuentran mayormente bajo un clima seco con temperaturas extremas, de lluvias estacionales y por lo general escasas. Hay estudios que señalan que las lluvias funcionan como factor condicionante para la infección por leptospira, aseverando que la ocurrencia de leptospirosis en alpacas se debe a una etiología multifactorial (Herrera et al., 2000). No obstante, la presencia de otras especies animales en el hábitat de los camélidos, podría contribuir a que la enfermedad se presente durante todo el año (Liceras de Hidalgo et al., 1989).

La ausencia de sueros positivos en animales jóvenes (tuis) no concuerda con lo esperado, pues diversos estudios en otras especies muestran no solo una mayor susceptibilidad en animales jóvenes, sino, además, una mayor severidad, que conduce a un menor desarrollo y una tasa de mortalidad variable (Radostits et al., 2002; Acha y Szyfres, 2003). Esta situación podría deberse a que en los otros estudios se consideró una mayor variedad de serovares.
Los hallazgos del presente estudio permiten incluir a los camélidos sudamericanos dentro de las especies susceptibles a infecciones por leptospira, más aún en una zona donde su crianza es de suma importancia para el poblador altoandino.

\section{Conclusiones}

- Se encontró una prevalencia de leptospirosis en alpacas de $44.8 \pm 5.3 \%$.

- Los serovares encontrados fueron pomona (43.6\%), icterohaemorrhagiae $(9.9 \%)$ y canicola $(1.5 \%)$.

- No se encontró una asociación entre el sexo y la infección con leptospira en la alpaca, ni se encontraron alpacas tuis seroreactoras.

\section{Literatura Citada}

1. Acha P, Szifres B. 2003. Zoonosis y enfermedades transmisibles comunes al hombre y a los animales. Vol I. $3^{\mathrm{a}}$ ed. Publicación científica y técnica $\mathrm{N}^{\circ} 850$. Washington, EEUU: Organización Panamericana de la Salud. 350 p.

2. Berquó E, Souza J, Gotlieb S. 1981. Bioestatística. Sao Paulo, Brasil: Ed. EPU. 350 p.

3. Brihuega B, Leoni L, Martínez $M$. 1996. Leptospirosis en llamas (Lama glama): Estudio serológico. Rev Arg Prod Anim 16: 393-396.

4. Daniel W. 1996. Bioestadística. $5^{\mathrm{a}} \mathrm{ed}$. México: Uteha. 876 p.

5. Elizalde A, Tenorio G, Velasco O. 2004. Identificación de Leptospira en la patogénesis de la uveítis crónica en la ciudad de México. Rev Mex Oftalmol 78(4): 164-170.

6. Herrera J, Vasconcellos $S$, Morais $Z$, Ferreira F, Sakamoto S, Ferreira J, Pinheiro S. 2000. Soropositividade para leptospirose em alpacas criadas no altiplano peruano. Puno, Peru. Análise de 
associação com o índice pluviometrico. Arq Inst Biol 67(2): 171-176.

7. Johnson LW. 1989. Llama reproduction. Vet Clin North Am: Food Anim Pract 5: 159-182.

8. Levett P. 2001. Leptospirosis. Clin Microbiol Rev 14: 296-326.

9. Liceras de Hidalgo J, Valdivia S, Higuchi E. 1989. Leptospirosis en el Perú. En: Análisis del seminario nacional de zoonosis y enfermedades de transmisión alimentaria. Lima: Ceres Ed. p. 7-20.

10. Ludeña H, Vargas A. 1982. Leptospirosis en alpacas. Avance Vet 2(2): 27-28.

11. Llorente P, Leoni L, Martínez M. 2002. Leptospirosis en camélidos sudamericanos. Estudio de prevalencia serológica en distintas regiones de la Argentina. Arch Med Vet 34(1): 59-68.

12. Macedo A, Hung A. 1993. Leptospirosis: Estudio serológico en alpacas (Lama pacos) de la SAIS Picotani - Puno. Rev Per Med Trop, UNMSM 7(2): 11-14.

13. Manual Merck de Veterinaria. 2000. $5^{\mathrm{a}}$ ed. Barcelona, España: Océano. 2558 p.

14. Martínez R, Obregón A, Pérez A, Baly A, Díaz, M, Baro M, Menéndez R, Ruiz A, Sierra G, López A. 1998. Reactogenicidad e inmunogenicidad de la primera vacuna cubana contra la leptospirosis humana. Rev Cub Med Trop 50(2): 159-166.

15. Myers DM. 1985. Leptospirosis. Manual de métodos para el diagnóstico de laboratorio. Nota Técnica $N^{\circ} 30$. Buenos Aires: Centro Panamericano de Zoonosis, OPS/OMS. $30 \mathrm{p}$.

16. Pérez C. 2000. Técnicas de muestreo estadístico. México:Ed. Alfaomega. 603 p.

17. Radostits M, Gay C, Blood D, Hinchcliff K. 2002. Medicina veterinaria: Tratado de enfermedades del ganado bovino, ovino, porcino, caprino y equino. Vol. I. $9^{a}$ ed. España: McGraw-Hill Interamericana. $1206 \mathrm{p}$.

18. Rosadio R, Rivera H, Chávez A, Serrano E, Quispe R, Rodríguez J, Yaya K. 2003. Seroprevalencia de agentes abortigénicos en alpacas de la provincia de Canchis, (Cuzco, Perú). En: III Congreso Mundial de Camélidos. Oruro, Bolivia. p 863-868.

19. Sentís H, Pardell H, Cobo E, Canela J. 1995. Bioestadística. $2^{a}$ ed. Barcelona, España: Ed. Masson. 305 p.

20. Sosa G, Santos O, Duarte C, Hernández D, Delgado L. 1988. Investigación serológica y bacteriológica de leptospirosis realizada en fauna exótica. Rev Cub Cienc Vet 19(3): 219-226.

21. Thiermann A. 1984. Leptospirosis: Current developments and trends. JAVMA 181: 722-725. 